\title{
FAR INFRARED LASER SPECTROSCOPY
}

L.-C. BRUNEL, A.L. BARRA, G. MARTINEZ and J.B. ROBERT

SNCI, CNRS, BP. 166X, F-38042 Grenoble cedex, France

\section{I - Introduction}

Far infrared laser spectroscopy developed after the first report of the production of far infrared (FIR) radiation by optical pumping ${ }^{1}$, is now widely used in plasma diagnostics, astronomy, metrology and solid state physics ${ }^{2}$. The advances in optically pumped FIR laser technology has been reviewed ${ }^{3}$. In the present paper we describe a laser which was designed for high stability.

\section{II - The Far infrared laser (Fig)}

The pump laser is a PL4 Edinburgh $\mathrm{CW} \mathrm{CO}_{2}$ 1aser, the far infrared resonator is home made. Depending upon the wavelength of interest we use two different geometries :

\section{i. Long wavelength range $(100 \mu \mathrm{m}-2 \mathrm{~mm})$}

The experimental set up is represented on the Fig, the resonator is made of two mirrors, the input concave mirror $M_{1}$ with a $3 \mathrm{~m}$ radius of curvature and a $1,5 \mathrm{~mm}$ diameter central hole, the output flat mirror $M_{2}$ with a central hole of 4 or $6 \mathrm{~mm}, M_{1}$ is fixed while $M_{2}$ is translated to tune the cavity length. The waveguide is a gold plated brass tube of $25 \mathrm{~mm}$ of inner diameter, with a length of $1.5 \mathrm{~m}$. The $\mathrm{CO}_{2}$ pump beam is focused into the resonator through a $\mathrm{Zn}$ Se Brewster window. Part of the FIR radiation is reflected by the Brewster window into a Golay detector. The signal from the Golay cell is used to stabilize the FIR output by monitoring the piezo tranducer of the $\mathrm{CO}_{2}$ laser.

\section{ii. Short wavelength range $(30-100 \mu \mathrm{m})$}

The mirror $M_{1}$ is a flat gold plated brass mirror with a $1.5 \mathrm{~mm}$ central hole, $M_{2}$ is a concave mirror with a $3 \mathrm{~m}$ radius of curvature, the output is 


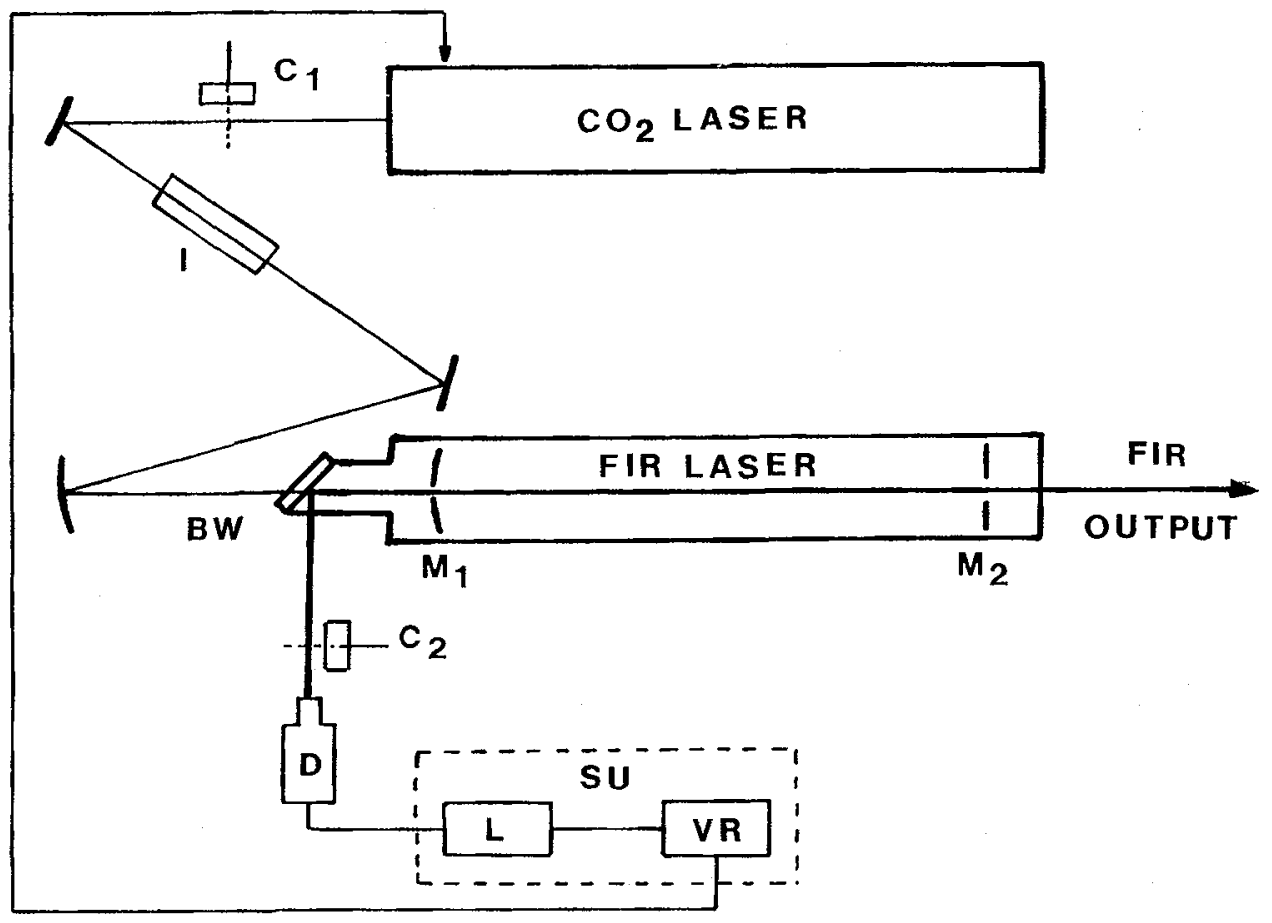

FAR INFRARED LASER

BW : Brewster Window ; $C_{1}, C_{2}$ : Chopper ; $D$ : Detector ; $M_{1}, M_{2}$ : Mirror ; I : Isolator ; L: Lock-in ; SU : Stabilisation Unit ; VR : Voltage Regulator

through $M_{1}$. The waveguide is a Pyrex tube of $17 \mathrm{~mm}$ of inner diameter with a length of $1.5 \mathrm{~m}$. The reference signal for the stabilisation unit is obtained by inserting a beam splitter on the output beam.

Whatever the geometry the stability of the laser is greatiy improve by using the passive isolator describe by Mansfeld et $a 7^{4}$. In order to use lock-in technique the output far infrared radiation is modulated by using the chopper $C_{1}$ or $C_{2}$.

\section{III - Applications}

This FIR laser was originally designed for magneto optical study of semiconductors ${ }^{5}$. It is currently used in either EPR spectroscopy at high frequency ${ }^{6}$ or in reflectivity measurements of high $T_{c}$ superconductors ${ }^{7}$. 


\section{REFERENCES}

1 - T.Y. Chang, T.J. Bridges

0pt. Commun. 1, 423 (1970)

2 - M.G. Douglas

Millimetre and submillimetre Wavelength Lasers, Springer Verlag (1989)

3 - D.T. Hodges Infrared Physics 18, 375 (1978)

4 - D.K. Mansfieid, A. Semet, L.C. Johnson

App 1. Phys. Lett. 37, 688 (1980)

5 - L.-C. Brune], S. Huant, M. Baj, W. Trzeciakowski

Phys. Rev. B33, 6863 (1986)

6 - L.-C. Brunel

International Symposium on Recent Advances in ESR spectroscopy Padova (1991)

in : Applied Magnetic Resonance, K.M. Shalikov ed. Springer Verlag

7 - G. Martinez, L.-C. Brunel, S. Louie, S.G. Labdi, H. Raffy International Conference on Materials and Mechanisms of Superconductivity, Kanazawa, Japon 1991 